\title{
CORRESPONDENCE
}

\section{An immunopathogenic perspective of interleukin-1 signaling}

\author{
Xinwen Lin ${ }^{1,2,3}$, Trix Twelkmeyer ${ }^{1,2}$, Si-Yu Wang ${ }^{4}$, Ruo-Nan Xu ${ }^{4}$, Fu-Sheng Wang (D) $^{4}$, Chao Zhang ${ }^{3,4}$ and Hong Tang ${ }^{1,2,5}$ \\ Cellular \& Molecular Immunology (2020) 17:892-893; https://doi.org/10.1038/s41423-020-0475-y
}

Interleukin-1 (IL-1), referred to as two distinct proteins, IL-1a and $\mathrm{IL}-1 \beta$, was first described almost 50 years ago. ${ }^{1}$ IL-1a and IL-1 $\beta$ represent immediate early innate cytokines critically involved in alarming and activating the host defense system. ${ }^{2}$ Therefore, any impairment of IL-1 signaling pathways often leads to devastating outcomes, such as autoimmunity and autoinflammation, dysmetabolism, cardiovascular disorders, and cancer. ${ }^{2}$ Many advances in targeting IL-1 in immune therapies have been achieved; for example, the IL-1-blocking agents anakinra (IL-1 receptor antagonist, IL-1Ra), canakinumab (anti-IL-1 $\beta$ mAb), and MABp1 (anti-IL-1a $\mathrm{mAb}$ ) have been approved for clinical use or are being evaluated. ${ }^{2}$ Remarkably, the CANTOS study, which included over 10,000 patients, showed that blocking IL-1 $\beta$ not only reduced atherosclerosis-related cardiovascular mortality but was also effective in inflammatory diseases related to lung cancer, arthrosis, and gout. ${ }^{3}$

Nevertheless, because of the specific spatiotemporal expression pattern of IL-1 and the complex regulatory networks of IL-1related pathways, it is still not fully understood how exactly IL-1 functions, and how to precisely rectify dysfunctional IL-1 signaling during diverse inflammatory conditions remains unknown.

For a long time, IL-1 $\alpha$ and IL-1 $\beta$, albeit sharing limited sequence homology, were considered redundant. They share similar threedimensional structures and interact with the same receptor, a heterodimer composed of IL-1R1 and IL-1R accessory protein (IL1Rap), to initiate the NF-KB signal transduction cascade. However, strong evidence is accumulating that IL-1 $\alpha$ and IL-1 $\beta$ each play specific roles in different pathological conditions (Table 1). For example, it was reported that neutrophil recruitment induced by necrotic cells is likely dependent on IL-1a but not IL-1 $\beta{ }^{4}$ The preferential usage of IL-1a over IL-1 $\beta$ for activating IL-1R1 has also been confirmed in other studies, including studies in druginduced liver injury (DILI), ${ }^{5}$ fatty acid-induced vascular response and atherosclerosis, ${ }^{6}$ and autoimmune disease. ${ }^{7}$ In a dextran sulfate sodium (DSS)-induced colitis mouse model, IL-1a from the intestinal epithelium drives intestinal inflammation, whereas IL-1 $\beta$ acts to heal the intestinal epithelial barrier. ${ }^{8}$ Moreover, in murine neonatal sepsis, IL-1 $\alpha$ but not IL-1 $\beta$ accounts for morbidity and mortality. ${ }^{9}$ IL-1a signaling is also critical in leukocyte recruitment and pulmonary inflammation in response to Aspergillus fumigatus $^{10}$ and Legionella pneumophila infection. ${ }^{11}$

IL-1 $\alpha$ and IL-1 $\beta$ differ from each other in gene expression and posttranslational modification. IL-1a precursor protein is expressed and preserved in a wild variety of mesenchymal cells, including keratinocytes, epithelial cells of the lung and entire gastrointestinal tract, and brain astrocytes. ${ }^{12}$ In contrast, the IL-1 $\beta$ precursor is an inducible factor produced mainly by myeloid cells after TLR signaling is activated. ${ }^{12}$ Furthermore, the IL-1 a precursor is fully active, and upon direct release from damaged cells, it functions as an alarmin to initiate the inflammatory response. IL1a precursor protein can also be cleaved by an array of different proteases, such as granzyme B, elastase, and calpain- 1 , leading to drastically enhanced bioactivity. The inactive IL-1 $\beta$ precursor, on the other hand, can be cleaved by inflammasome-activated caspase- 1 and released via a tightly controlled GSDMD pore to the extracellular matrix. ${ }^{13}$ It is worth noting that most studies on inflammasomes or IL-1 $\beta$ do not exclude the potential involvement of IL-1a, especially considering that inflammasomal activation also facilitates IL-1a secretion. ${ }^{14}$

The understanding of IL-1 $a$ and IL-1 $\beta$ is also complicated due to their shared usage of IL-1R1, which uses MyD88 as an adaptor in the pro-inflammatory NF-KB signaling pathway. IL-1R1 signal specificity may be based on the IL-1R1-expressing cell type and associated IL-1 stimulation from neighboring cells. In a mouse model of DILI, the expression of IL-1R1 is mainly restricted to myeloid cells among hepatic lymphocytes. In one study, IL-1a made by macrophages activated neutrophils via a paracrine loop and promoted hepatic injury during the early phase of DILI. ${ }^{5}$ In another study, liver cells lacking IL-1R1 resisted cell death but were dependent on neighboring cells, arguing for the involvement of IL-1 from these cells. ${ }^{15}$ The involvement of IL-1 in distinct immunological, neural, and physiological activities in the brain has recently been revealed in vivo, and it depended on different cell type-specific IL-1R1 signaling pathways. Liu et al. employed genetic knock-in reporter mice to track and reciprocally delete and/or express IL-1R1 in specific CNS cell types, including endothelial cells, ventricular cells, peripheral myeloid cells, microglia, astrocytes, and neurons. Particularly, they demonstrated that endothelial IL-1R1-driven leukocyte recruitment to the central nervous system accounted for impaired neurogenesis; ventricular IL-1R1 regulated monocyte recruitment; and noninflammatory ventricular, astrocyte, and neuronal IL-1R1-mediated neuromodulatory activities. ${ }^{16,17}$ In addition, IL-1 is also a licensing signal to permit effector cytokine production by precommitted $\mathrm{T}$ helper lineage cells, including Th1, Th2, and Th17 cells. IL-1R signaling stabilizes cytokine transcripts to enable productive and rapid effector functions in CD4+ T cells. ${ }^{18}$ Moreover, the pathogenetic roles of GM-CSF-secreting Th cells have been reported in central nervous system inflammation, ${ }^{19}$ sepsis, $^{20}$ and the recently reported COVID-19. ${ }^{21}$ IL-1R signaling is required for the maintenance and pathogenicity of GM-CSF-producing Th cells. ${ }^{22}$ Specifying the cell sources and magnitude of IL-1a and IL-1 $\beta$

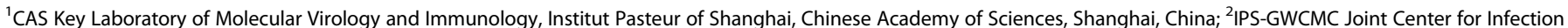

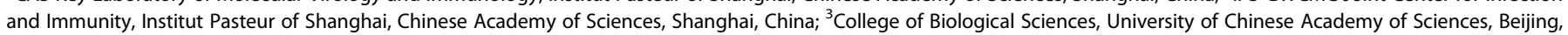

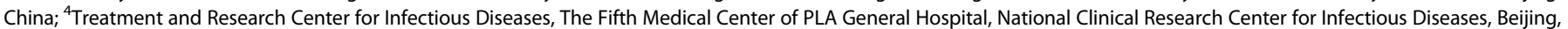
China and ${ }^{5}$ Pasteurien College, Suzhou University, Jiangsu, China

Correspondence: Chao Zhang (zhangch302@163.com) or Hong Tang (htang@ips.ac.cn)

Received: 9 May 2020 Accepted: 14 May 2020

Published online: 28 May 2020 


\begin{tabular}{|c|c|c|c|}
\hline Chen et al. ${ }^{4}$ & $\begin{array}{l}\text { Cell death induced } \\
\text { inflammation }\end{array}$ & Macrophage & $\begin{array}{l}\text { Cell death-triggered inflammation required IL- } 1 \alpha \text {, and IL-1R function on } \\
\text { non-bone marrow-derived cells was required. }\end{array}$ \\
\hline Zhang et al. ${ }^{5}$ & DILI & Macrophage & $\begin{array}{l}\text { IL- } 1 \alpha \text {, rather than IL- } 1 \beta \text {, was critically involved in the immunopathogenesis } \\
\text { of AlLI. Activation of IL-1 } \alpha \text { depended on Kupffer cells that sense and } \\
\text { transduce DAMP signaling through the TLR4/MyD } 88 \text { pathway. }\end{array}$ \\
\hline Lukens et al. ${ }^{7}$ & Autoinflammation & Hematopoietic cells & $\begin{array}{l}\text { IL- } 1 \alpha \text {, but not IL- } 1 \beta \text { or RIP3-mediated necroptosis, was critical for } \\
\text { exacerbated inflammatory responses and unremitting tissue damage upon } \\
\text { footpad microabrasion of Ptpn6 } 6^{\text {spin }} \text { mice. }\end{array}$ \\
\hline Bersudsky et al. ${ }^{8}$ & $\begin{array}{l}\text { DSS-induced colon } \\
\text { inflammation }\end{array}$ & $\begin{array}{l}\text { Intestinal epithelial } \\
\text { cells (IECs) }\end{array}$ & $\begin{array}{l}\text { The roles of IL-1 } \alpha \text { and IL-1 } \beta \text { differed in DSS-induced colitis in that IL-1 } \alpha \text {, } \\
\text { mainly expressed by colon epithelial cells, was inflammatory, whereas IL-1 } 1 \beta \text {, } \\
\text { mainly of myeloid cell origin, promoted healing and repair. }\end{array}$ \\
\hline Barry et al. ${ }^{11}$ & $\begin{array}{l}\text { Legionella pneumophila } \\
\text { infection }\end{array}$ & Hematopoietic cells & $\begin{array}{l}\text { IL-1 } \alpha \text { was a critical initiator of neutrophil recruitment to the lungs of } L \text {. } \\
\text { pneumophila-infected mice. }\end{array}$ \\
\hline
\end{tabular}

signaling through the shared IL-1R1 is critical to understanding CD4+ T helper functions.

The therapeutic activities of anti-IL-1 antibodies across diseases argue for innate inflammatory response as a metanarrative in modern medicine. More efforts are needed to clarify the roles of IL-1/IL-1R1 signaling and effectors to better understand the immunopathogenesis of diseases and improve current targeted treatments.

\section{ADDITIONAL INFORMATION}

Competing interests: The authors declare no competing interests.

\section{REFERENCES}

1. Mizel, S. B. \& Farrar, J. J. Revised nomenclature for antigen-nonspecific T-cell proliferation and helper factors. Cell. Immunol. 48, 433-436 (1979).

2. Mantovani, A., Dinarello, C. A., Molgora, M. \& Garlanda, C. Interleukin-1 and related cytokines in the regulation of inflammation and immunity. Immunity 50, 778-795 (2019)

3. Ridker, P. M. et al. Modulation of the interleukin- 6 signalling pathway and incidence rates of atherosclerotic events and all-cause mortality: analyses from the Canakinumab Anti-Inflammatory Thrombosis Outcomes Study (CANTOS). Eur. Heart J. 39, 3499-3507 (2018).

4. Chen, C.-J. et al. Identification of a key pathway required for the sterile inflammatory response triggered by dying cells. Nat. Med. 13, 851-856 (2007).

5. Zhang, C. et al. Macrophage-derived IL-1alpha promotes sterile inflammation in a mouse model of acetaminophen hepatotoxicity. Cell Mol. Immunol. 15, 973-982 (2018).

6. Freigang, S. et al. Fatty acid-induced mitochondrial uncoupling elicits inflammasome-independent IL-1alpha and sterile vascular inflammation in atherosclerosis. Nat. Immunol. 14, 1045-1053 (2013).

7. Lukens, J. R. et al. RIP1-driven autoinflammation targets IL-1a independently of inflammasomes and RIP3. Nature 498, 224-227 (2013).
8. Bersudsky, M. et al. Non-redundant properties of IL-1a and IL-1 $\beta$ during acute colon inflammation in mice. Gut 63, 598-609 (2014).

9. Benjamin, J. T. et al. Cutting Edge: IL-1 $a$ and not IL-1 $\beta$ drives IL-1R1-dependent neonatal murine sepsis lethality. J. Immunol. 201, 2873-2878 (2018).

10. Caffrey, A. K. et al. IL-1alpha signaling is critical for leukocyte recruitment after pulmonary Aspergillus fumigatus challenge. PLoS Pathog. 11, e1004625 (2015).

11. Barry, K. C., Fontana, M. F., Portman, J. L., Dugan, A. S. \& Vance, R. E. IL-1a signaling initiates the inflammatory response to virulent Legionella pneumophila in vivo. J. Immunol. 190, 6329-6339 (2013).

12. Malik, A. \& Kanneganti, T. D. Function and regulation of IL-1alpha in inflammatory diseases and cancer. Immunol. Rev. 281, 124-137 (2018).

13. Broz, P., Pelegrin, P. \& Shao, F. The gasdermins, a protein family executing cell death and inflammation. Nat. Rev. Immunol. 20, 143-157 (2020).

14. Groß, O. et al. Inflammasome activators induce interleukin-1a secretion via distinct pathways with differential requirement for the protease function of caspase1. Immunity 36, 388-400 (2012).

15. Gehrke, N. et al. Hepatocyte-specific deletion of IL1-RI attenuates liver injury by blocking IL-1 driven autoinflammation. J. Hepatol. 68, 986-995 (2018).

16. Liu, X. et al. Cell-type-specific interleukin 1 receptor 1 signaling in the brain regulates distinct neuroimmune activities. Immunity 50, 317-333 e316 (2019).

17. Visan, I. Mapping IL-1 in the brain. Nat. Immunol. 20, 245 (2019).

18. Jain, A., Song, R., Wakeland, E. K. \& Pasare, C. T cell-intrinsic IL-1R signaling licenses effector cytokine production by memory CD4 T cells. Nat. Commun. 9, 3185 (2018).

19. Stienne, C. et al. Foxo3 transcription factor drives pathogenic $T$ helper 1 differentiation by inducing the expression of eomes. Immunity 45, 774-787 (2016).

20. Huang, $H$. et al. High levels of circulating GM-CSF $+C D 4+T$ cells are predictive of poor outcomes in sepsis patients: a prospective cohort study. Cell. Mol. Immunol. 16, 602-610 (2019).

21. Zhou, Y. et al. Pathogenic T cells and inflammatory monocytes incite inflammatory storm in severe COVID-19 patients. Nat. Sci. Rev. https://doi.org/10.1093/nsr/ nwaa041 (2020).

22. Komuczki, J. et al. Fate-mapping of GM-CSF expression identifies a discrete subset of inflammation-driving T helper cells regulated by cytokines IL-23 and IL1ß. Immunity 50, 1289-1304. e1286 (2019). 